\title{
Characterisation of the dynamical quantum state of a zero temperature Bose-Einstein condensate
}

\author{
J. Rogel-Salazar ${ }^{\mathrm{a}, 1}$ S. Choi ${ }^{\mathrm{b}}$ G.H.C. New ${ }^{\mathrm{a}}$ K. Burnett ${ }^{\mathrm{c}}$ \\ ${ }^{a}$ Quantum Optics 8 Laser Science Group, Department of Physics, Imperial \\ College, London $S W^{\prime}$ 2BW, U.K. \\ ${ }^{\mathrm{b}}$ Department of Chemistry, University of Rochester, P.O. RC Box 270216, \\ Rochester, New York 14627-0216 \\ ${ }^{\mathrm{c}}$ Clarendon Laboratory, Department of Physics, University of Oxford, Parks Road, \\ Oxford OX1 3PU, U.K.
}

\begin{abstract}
We describe the quantum state of a Bose-Einstein condensate at zero temperature. By evaluating the $Q$-function we show that the ground state of Bose-Einstein condensate under the Hartree approximation is squeezed. We find that multimode Schrödinger cat states are generated as the condensate evolves in a ballistic expansion.
\end{abstract}

Key words: Bose-Einstein condensation, Quantum state, Squeezing, Schrödinger cat states

PACS: 03.75.Fi, 03.65.Wj, 42.50.Dv

\section{Introduction}

The realisation of Bose-Einstein condensation in dilute gases has made it possible to study the dynamics of quantum fields directly [1,2]. In this paper, we address one of the most intriguing issues surrounding a Bose-Einstein condensate (BEC), namely the nature of its quantum state. Number states might seem to be the natural choice given that atoms cannot be created or destroyed

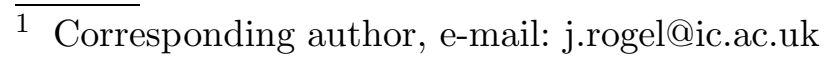


at these energies. However, it is well known that, in an open environment, particles may be added or removed from the condensate, and this implies that the condensate is not in a pure Fock state. On the other hand, it has been suggested that there should be a highly entangled state of the condensate plus the environment [3]. The coherence properties of the condensate have been demonstrated experimentally, indicating that a coherent state might be the most robust representation $[4,5,6]$. This point turns out to be very important, as it is well-known that a coherent state propagating through an amplitudedispersive medium evolves into a superposition of two coherent states [7]. The generation of these Schrödinger cat-like states is considered to be a general property of nonlinear systems where dissipation effects are not very large. Recently, some schemes to create Schrödinger cat states in BECs have been proposed [8] and the effects of loss have also been considered.

It has also been pointed out that the state of the condensate might be squeezed, given that binary collisions between atoms play a very important role in the description of BECs [9] and are known to give rise to squeezing [10,11,12]. We note that the question of describing the quantum state of a trapped BEC was addressed in reference [11] where a single mode approximation was considered, based on a symmetry-breaking picture. The authors showed that for repulsive interactions the state is number squeezed. In this paper, we use a multimode description in the Hartree approximation. In particular, we show how the state evolves under ballistic expansion, which is important as this is the basis of many experiments involving the condensates $[4,5,6]$.

To study the evolution of the quantum state of a BEC, we have used the Heisenberg equation of motion obtained from the many-body Hamiltonian describing the condensate, and used the Hartree approximation, under which the $n$-atom wavefunction is written as a product of $n$ single atom wavefunctions. This results in a nonlinear Schrödinger equation for the condensate wavefunction, or the well-known Gross-Pitaevskii equation (GPE). The GPE has proved to be a very useful tool for studying the condensate dynamics, and its predictions are in very good agreement with experimental results at low temperatures $[13,14]$. It is important to note that the GPE can be understood in terms of either coherent states or number states. Here, we make use of a number state description in order to obtain an analogue of the scenario presented in [15] for the case of light. We shall take "snapshots" of the wavefunction at various times and analyse them using the $Q$-function, which is a quantum-mechanical phase space function. We also want to mention that we are treating the condensate in a zero temperature regime; this means that we are basically referring to the predominant mechanisms occurring in the ground state of the condensate. At finite temperature, the mechanism is affected by the presence of excited states and therefore the dynamics are changed [16].

The paper is organised as follows: in Section 2, we present the Hamiltonian 
that describes the BEC and solve the equation of motion in the Schrödinger picture using the Hartree approximation. In Section 3, we calculate the $Q$ function for the condensate. The evolution of the state of the condensate in a ballistic expansion is then presented, and the generation of Schrödinger catlike states is observed. This is relevant in studies of output couplers or atom lasers. Finally we conclude in Section 4.

\section{Q-Function in the Hartree approximation}

The phase space representation is useful for visualising the evolution of a quantum state, as the statistics of the state can easily be described using the quasiprobability distribution functions [17]. In this case, we have made use of the $Q$-function which has the nice property of being always positive. This function can be considered to describe probability densities, and has the further advantage of being readily measurable by quantum tomographic techniques $[12,18]$. The first experimental measurement of quantum states was reported by Smithey et. al. in 1993 [19]. Since then, several proposals to reconstruct the quasiprobability distributions of a quantum system, such as the Wigner function, have been made. For instance, Kurtsiefer et. al. [20] reported a technique to measure the Wigner function of an ensemble of helium atoms in a double-slit experiment. Moreover, a proposal has been made to measure the quantum state of a BEC in an atomic interferometer based on Raman transitions [12], where the $Q$-function can be measured directly. The way of obtaining the quasiprobability function is analogous to doing unbalanced homodyne detection of an optical signal.

The $Q$-function of a pure quantum state $|\Psi\rangle$ is defined as

$$
Q\left(\alpha_{r}, \alpha_{i}\right) \equiv \frac{1}{\pi}|\langle\alpha \mid \Psi\rangle|^{2}
$$

where the two real numbers $\alpha_{r}$ and $\alpha_{i}$ describe the coherent state $|\alpha\rangle$. Therefore, the quasiprobability distribution depends directly on these two parameters, which span the phase space. An alternative form of equation (1), leads the $Q$-function to be defined by the diagonal matrix elements of a density operator $\hat{\rho}$ in a pure coherent state $|\alpha\rangle$ :

$$
Q\left(\alpha_{r}, \alpha_{i}\right) \equiv \frac{1}{\pi}\langle\alpha|\hat{\rho}| \alpha\rangle
$$

Clearly, the $Q$-function is characterised by being always positive and normalised to unity. Thus, it can be regarded as describing probability densities, 
and the numbers $\alpha_{r}$ and $\alpha_{i}$ play the role of conjugate variables, eg number and phase.

We shall start by describing the system in terms of the many-body interacting Hamiltonian

$$
\begin{aligned}
\hat{H}= & \int \mathrm{d}^{3} \mathbf{r} \hat{\phi}^{\dagger}(\mathbf{r}, \tau)\left[-\frac{\hbar^{2}}{2 m} \nabla_{\mathbf{r}}^{2}+V_{\text {trap }}(\mathbf{r})\right] \hat{\phi}(\mathbf{r}, \tau) \\
& +\frac{1}{2} \int \mathrm{d}^{3} \mathbf{r} \mathrm{d}^{3} \mathbf{r}^{\prime} \hat{\phi}^{\dagger}(\mathbf{r}, \tau) \hat{\phi}^{\dagger}\left(\mathbf{r}^{\prime}, \tau\right) \hat{V}\left(\mathbf{r}-\mathbf{r}^{\prime}\right) \hat{\phi}(\mathbf{r}, \tau) \hat{\phi}\left(\mathbf{r}^{\prime}, \tau\right),
\end{aligned}
$$

where $\hat{\phi}^{\dagger}(\mathbf{r}, \tau)$ and $\hat{\phi}(\mathbf{r}, \tau)$ are the boson field creation and annihilation operators respectively, and $\hat{V}\left(\mathbf{r}-\mathbf{r}^{\prime}\right)$ is the two-body interatomic potential. For a contact interaction potential, the normalised Heisenberg equation of motion for the operator $\hat{\phi}$ is given by

$$
\mathrm{i} \frac{\partial \hat{\phi}}{\partial t}=\left[-\nabla_{\mathbf{r}}^{2}+V_{\text {trap }}(\mathbf{r})+\frac{n U_{0}}{\hbar \omega_{\text {trap }}} \hat{\phi}^{\dagger} \hat{\phi}\right] \hat{\phi}
$$

In the Schrödinger picture, equation (5) takes the form $\mathrm{i} \hbar \frac{\mathrm{d}}{\mathrm{d} t}|\Phi\rangle=\hat{H}|\Phi\rangle$. The solution can then be found by expanding the state vector $|\Phi\rangle$ in a Fock space

$$
|\Phi\rangle=\sum_{n} A_{n}|n ; t\rangle
$$

The solutions of the equation are then number states $|n ; t\rangle$. In the Hartree approximation, it is possible to write the number states as

$$
|n ; t\rangle=\frac{1}{\sqrt{n !}}\left(\int \mathrm{d} x \Psi_{n}(x, t) \hat{\phi}^{\dagger}(x)\right)^{n}|0\rangle,
$$

where $\Psi_{n}$ is a single particle wavefunction and $\hat{\phi}^{\dagger}$ corresponds to the creation operator of the field.

This implies that each particle in the system experiences the same potential. Therefore, the single-particle function $\Psi_{n}$ satisfies the GPE with a scaled nonlinearity proportional to the number of particles [15].

If we superimpose these states with a Poissonian distribution, the coefficient $A_{n}$ takes the form

$$
A_{n}=\frac{\alpha_{0}^{n}}{\sqrt{n !}} \exp \frac{-\left|\alpha_{0}\right|^{2}}{2}
$$


so that the quantum state in the Hartree approximation is expressed as

$$
|\Phi\rangle=\sum_{n} \frac{\alpha_{0}^{n}}{n !} \exp \frac{-\left|\alpha_{0}\right|^{2}}{2}\left(\int \mathrm{d} x \Psi_{n}(x, t) \hat{\phi}^{\dagger}(x)\right)^{n}|0\rangle .
$$

The $Q$-function given by equation (1) can be written in terms of a reference coherent state $|\alpha,\{\Psi(x, t)\}\rangle$ as

$$
Q\left(\alpha_{r}, \alpha_{i}\right)=|\langle\alpha,\{\Psi(x, t)\} \mid \Phi\rangle|^{2}
$$

We can construct the reference coherent state $|\alpha,\{\Psi(x, t)\}\rangle$ using the $n$ particle eigenstate for the field with envelope $\Psi_{\bar{n}}(x, t)$, where $\bar{n}=\left|\alpha_{0}\right|^{2}$ has the meaning of the average particle number

$$
\left|n,\left\{\Psi_{\bar{n}}(x, t)\right\}\right\rangle=\frac{1}{\sqrt{n !}}\left(\int \mathrm{d} x \Psi_{\bar{n}}(x, t) \hat{\phi}^{\dagger}(x)\right)^{n}|0\rangle .
$$

Assuming Poissonian statistics, we obtain the many-body coherent state

$$
\left|\alpha,\left\{\Psi_{\bar{n}}(x, t)\right\}\right\rangle=\exp \left(\frac{-|\alpha|^{2}}{2}\right) \exp \left(\alpha \int \mathrm{d} x \Psi_{\bar{n}}(x, t) \hat{\phi}^{\dagger}(x)\right)|0\rangle .
$$

Using equations (9), (10) and (12), the $Q$-function can be written

$$
Q\left(\alpha_{r}, \alpha_{i}, t\right)=\exp \left(-\left|\alpha_{0}\right|^{2}-|\alpha|^{2}\right) \times\left|\sum_{n=0}^{\infty} \frac{\left(\alpha^{*} \alpha_{0}\right)^{n}}{n !}\left(\int \mathrm{d} x \Psi_{\bar{n}}^{*}(x, t) \Psi_{n}(x, t)\right)^{n}\right|^{2}
$$

\section{Quantum state of a Bose-Einstein Condensate at zero tempera- ture}

Let us consider Gaussian wavefunctions to describe the ground state of the trapped condensate

$$
\Psi_{n}=\exp \left[-\left(\frac{x}{n-1}\right)^{2}\right]
$$

and assume that $\bar{n}$ is large so that the difference between $\bar{n}-1$ and $\bar{n}$ can be neglected. The $Q$-function for such a case is

$$
Q_{G}\left(\alpha_{r}, \alpha_{i}\right)=\exp \left(-\left|\alpha_{0}\right|^{2}-|\alpha|^{2}\right) \times\left|\sum_{n=2}^{\infty} \frac{\left(\alpha^{*} \alpha_{0}\right)^{n}}{n !}\left[\sqrt{\pi}\left(\frac{\bar{n}^{2} n^{2}}{n^{2}+\bar{n}^{2}}\right)\right]^{n}\right|^{2}
$$


For an average number of particles $\bar{n}=50$ the $Q$-function (15) is shown in Figure 1.

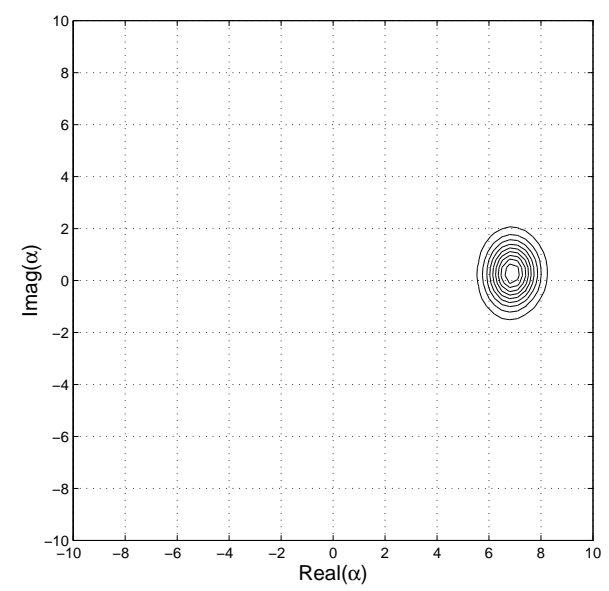

Fig. 1. Q-function for a Bose condensed gas described by Gaussian functions.

A more realistic approximation to the ground state is given by the ThomasFermi solution

$$
\Psi_{n}=\frac{1}{\sqrt{n(n-1) U_{0}}}\left(\mu_{n}-V_{\text {trap }}\right)^{1 / 2},
$$

where the chemical potential $\mu_{n}$ is determined by the normalisation of $\Psi_{n}$. The $Q$-function is then expressed as

$$
\begin{aligned}
Q_{T F}\left(\alpha_{r}, \alpha_{i}\right)= & \exp \left(-\left|\alpha_{0}\right|^{2}-|\alpha|^{2}\right) \times \\
& \mid \sum_{n=2}^{\infty} \frac{\left(\alpha^{*} \alpha_{0}\right)^{n}}{n !}\left\{\frac { 4 } { 3 } \mu _ { n } \left[\left(\mu_{n}-\mu_{\bar{n}}\right) \mathrm{F}\left(\sqrt{\frac{\mu_{n}}{\mu_{\bar{n}}}}, \sqrt{\frac{\mu_{\bar{n}}}{\mu_{n}}}\right)-\right.\right. \\
& \left.\left.\left(\mu_{n}+\mu_{\bar{n}}\right) \mathrm{E}\left(\sqrt{\frac{\mu_{n}}{\mu_{\bar{n}}}}, \sqrt{\frac{\mu_{\bar{n}}}{\mu_{n}}}\right)\right]\right\}\left.^{n}\right|^{2},
\end{aligned}
$$

where $\mathrm{F}(\varphi, k)$ and $\mathrm{E}(\varphi, k)$ are incomplete elliptic integrals of the first and second kind respectively [21]. For a harmonic trap potential $V_{1}=\frac{1}{2} m \omega_{\text {trap }}^{2} r^{2}$, the chemical potential is given by $\mu_{n}=\left(\frac{3 n(n-1) U_{0}}{8}\right)^{2 / 3}$. The $Q$-function for the same average number of particles as in the previous case is plotted in Figure 2 .

From Figures 1 and 2, it is clear that the ground state of the condensate is very close to a squeezed state, since the $Q$-function has different widths in the $\alpha_{r}$ and $\alpha_{i}$-directions. On the one hand, the squeezing is generated by the nonlinearity inherent to the system. This can be explained in terms of a 


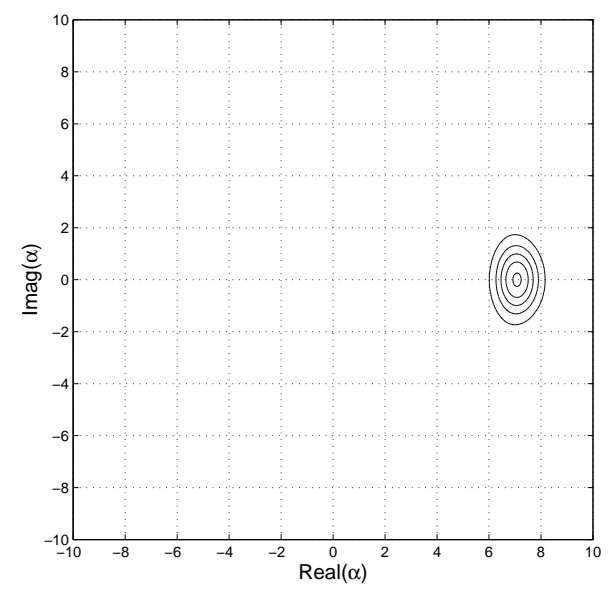

Fig. 2. $Q$-function for a Bose condensed gas in the Thomas-Fermi approximation.

superposition of different numbers of particles with different phases, creating a deviation from the classical phase. On the other hand, we know that the coherent state interpretation arises from the robustness of these states in the presence of interactions between the condensate and its environment [3]. It is well known that coherent states are a particular case of a more general class of minimum-uncertainty states, namely the squeezed states. It has been shown that the propagation of a coherent state in a nonlinear medium evolves into a superposition of two coherent states [7], while the quantum-mechanical superposition of two coherent states has been shown to lead to a transition from a Poisson distribution to a sub-Poissonian one and squeezing [22]. In this sense, the robust description of the quantum state still applies, bearing in mind that the existence of the interactions between atoms yields squeezing of the quantum field and, as a result, entanglement between the atoms is also produced [9]. We note that while a single coherent state does not evidence squeezing, a considerable amount is exhibited in a superposition of even just two coherent states.

This mechanism of achieving squeezing is the predominant one in the case of the ground state, without the presence of highly populated excited states. When we consider the presence of a thermal cloud, we can treat the system using the Bogoliubov procedure and analyse the behaviour of the collective excitations. It has been shown that squeezed quasiparticle excitations can be produced when considering the generation of correlated pairs by the interaction of an excited state with the ground state [16]. Thus, we can talk about two different aspects of nonclassicality in the system, the predominant one for the ground state at zero temperature, and the prevalent one in the presence of excited states.

It is of particular interest to see how the $Q$-function behaves once the condensate is released from the trap as when the atomic cloud is allowed to expand ballistically [1], or in some output coupling schemes for atom lasers 
[23]. The Thomas-Fermi solution was used as an initial condition to solve the GPE after the trapping potential is switched off and the $Q$-function (17) was then calculated from the dynamical evolution of the wavefunctions. We expect that once the trapping potential is switched off, the condensate will rethermalise. Comparing the quantum state of the condensate, for a trap frequency $w_{\text {trap }}=2 \pi \times 100 \mathrm{~Hz}$, at $2 \times 10^{-3} \mathrm{~s}$ (shown in Figure 3 ) to the $Q$-function for a thermal state (Figure 4) makes it clear that rethermalisation is indeed taking place.

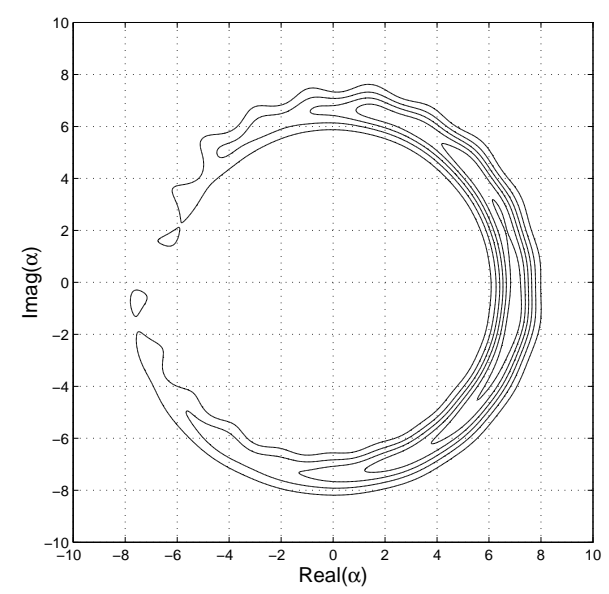

Fig. 3. $Q$-function for the dynamical evolution of the system at $2 \times 10^{-3} \mathrm{~s}$.

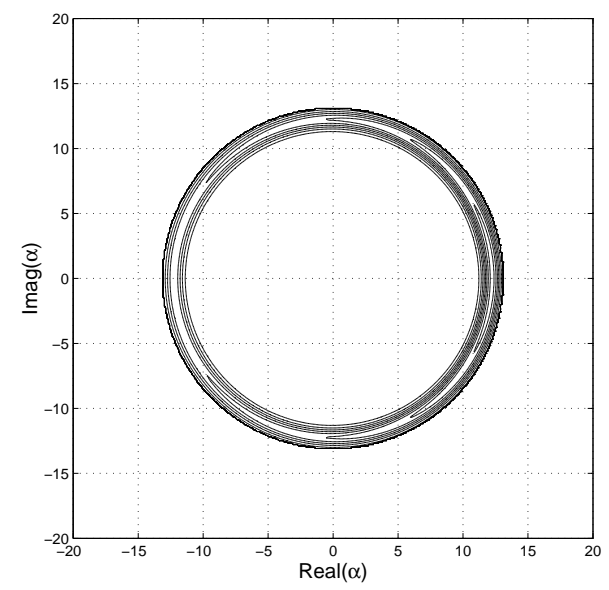

Fig. 4. $Q$-function for a typical thermal state

A very important aspect that has to be considered is the finite lifetime of the condensate, which is at most 10-20 s. The reason behind this, is that the thermal cloud is not the only source of decoherence. The condensate loses atoms because of Rayleigh scattering, external heating, and three-body decay. Information about the quantum state of the condensate is carried by the atoms that escape from it so that, due to interactions of the system with the environment, a quantum superposition is turned into a statistical mixture. From the processes mentioned above, three-body recombination is the most 
important one [24,25]. In an experiment carried out by Stamper-Kurn et. al. [26], the measured loss rate per atom turned out to be $4 / s$ for $N=10^{7}$ atoms. This rate scales as $N^{3}$, where $N$ is the number of atoms in the condensate. Considering a condensate with $N=10^{4}$, just one atom is lost per second; the decoherence time is $1 \mathrm{~s}$. Bearing this fact in mind, it is perfectly sensible to ask whether, after opening the trap, it is still possible to see a Schrödinger cat-like state. When the states evolve further, a multi-component structure develops as shown in Figures 5 and 6 , at $5 \times 10^{-3} \mathrm{~s}$ and $1 \times 10^{-2} \mathrm{~s}$, respectively. In both figures, the quasiprobability function demonstrates that the quantum states obtained correspond to Schrödinger cat states like the ones generated in Kerr media $[27,28]$. Moreover, we also see that the cat states have a different number of components at different times in the evolution, in a close analogy to the mechanism discussed by Yao for the case of a radiation field propagating in a nonlinear medium [29]. It has been shown that the quantum-mechanical superposition of states can yield a state that exhibits squeezed fluctuations $[30,22]$. This suggests that, even in the presence of losses due to three-body recombination, it would still be possible to observe Schrödinger cat-like states.

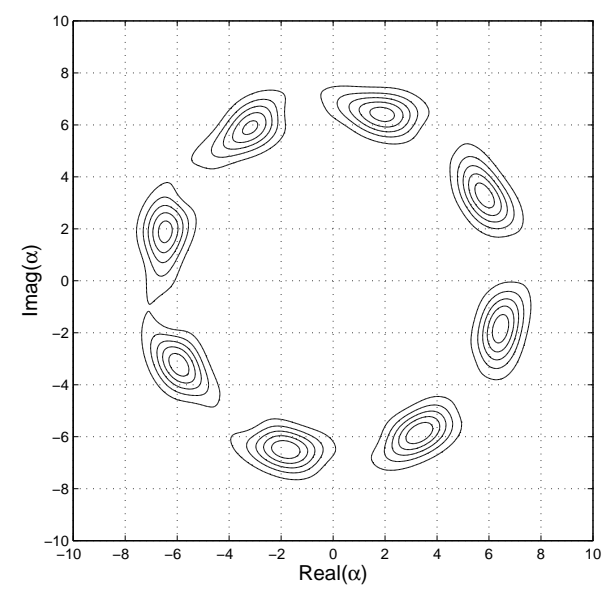

Fig. 5. $Q$-function for the dynamical evolution of the system at $5 \times 10^{-3} \mathrm{~s}$.

\section{Discussion}

In summary, we have described the ground state of a Bose condensed gas in the Hartree approximation in a phase-space representation using the $Q$ function. We have confirmed that the ground state of the condensate is in a squeezed state due to the presence of interactions between the atoms. We have also studied the dynamical evolution of the condensate in a ballistic expansion. The simulations show that immediately after the condensate is released from the trap the system rethermalises, with the quantum state being 


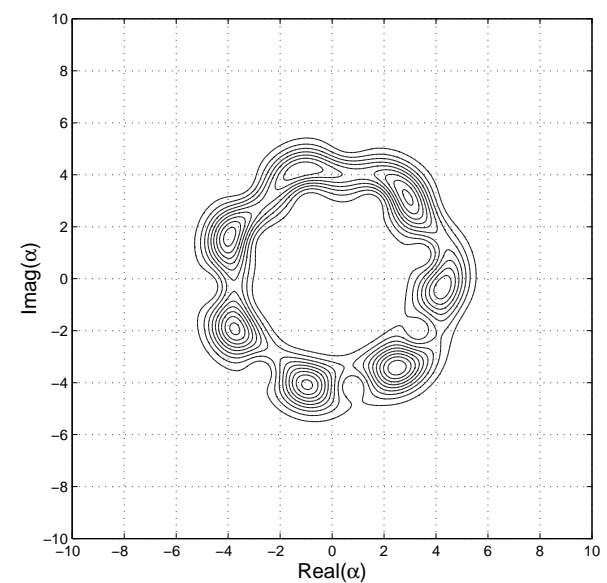

Fig. 6. $Q$-function for the dynamical evolution of the system at $1 \times 10^{-2}$ s.

still close to a squeezed state. Continuing the evolution without the trapping potential, we obtain a multimode Schrödinger cat-like structure. In general, the "longevity" of these states is very small due to decoherence. However, the time scales of the calculations suggest that it might still be possible to observe them. The generation of nonclassical states by the time evolution of an initial coherent state under the influence of some nonlinear dynamics has been studied in the past for the case of radiation fields $[7,27,28,29]$. In the case of a BEC the nonlinearity arises from binary correlations between the atoms, producing the effective interactions between them. We point out that this process basically refers to the ground state, as the presence of excited states changes the predominant mechanisms of achieving squeezing.

An issue that remains to be addressed is the one related to the experimental reconstruction of the quantum state of the condensate. We have mentioned that it is possible to use quantum tomographic techniques to measure the $Q$-function $[12,18]$. As an alternative to these techniques, a scheme using projection synthesis was proposed by Baseia and co-workers [31]. The scheme follows the idea of measuring the probability distribution of a state $|f\rangle$ to have an observable $\theta$

$$
\langle f \mid \theta\rangle\langle\theta \mid f\rangle=P_{N}(\theta)=\frac{1}{2 \pi}\left|\sum_{n=0}^{N} c_{n} \exp (-\mathrm{i} n \theta)\right|^{2},
$$

where the expectation value is replaced with the probability density by setting $c_{n}$ to zero for $n>N$, with large $N$. The probability density is proportional to the expectation value of the projector $\hat{\pi}=\kappa|\theta\rangle\langle\theta|$ where $\kappa$ is a positive constant. If the field is in a mixed state $\hat{\rho}$, equation (18) can be written as

$$
P_{\rho}(\theta)=\operatorname{Tr}(\hat{\rho} \hat{\pi})=\langle\theta|\hat{\rho}| \theta\rangle
$$


It has been shown [32] that by specifying a suitable reference state $|B\rangle_{b}$ in an experimental setup where a state $|\psi\rangle_{a}$ is coherently mixed in a beam splitter with the reference state $|B\rangle_{b}$, we can obtain the required probability (19).

In the case of the quasiprobability function $Q$, we would require a projector $\hat{\pi}=\kappa|\alpha\rangle\langle\alpha|$ so that its substitution in equation (19) yields

$$
P_{\rho}(\alpha)=\operatorname{Tr}(\hat{\rho} \hat{\pi})=\langle\alpha|\hat{\rho}| \alpha\rangle=Q\left(\alpha_{r}, \alpha_{i}\right)
$$

The form of the reference state that leads to the required projector is given by [31]

$$
|B\rangle_{b}=C \sum_{k=0}^{N} b_{k}|k\rangle_{b}
$$

where $C$ is a normalisation constant and

$$
b_{k}=\frac{2^{-N}}{C}\left(\begin{array}{c}
N \\
k
\end{array}\right)^{-1 / 2} \exp \left(\frac{\mathrm{i} k \pi}{2}\right) C_{N-k}^{*} .
$$

It is important to mention that this scheme involves projections onto truncated coherent states, which is a good approximation for large $N$. In other words, it works well for the case $N \gg \bar{n}$, where $\bar{n}$ is the average particle number of the synthesised coherent state. The construction of the reference state (21) would be the main experimental challenge in this scheme. However, one might able to generate such a state using the scheme proposed in [33] where the authors show that it is possible to prepare an arbitrary (finite) superposition of Fock states by applying a well-defined succession of the displacement and creation operators. Experimentally a sequence of beam splitters at which conditional measurements are performed is required. In the case of BEC, this may be achieved by using repeated interference of condensates with different hyperfine states, similar to the Gedanken experiment proposed by Castin and Dalibard in reference [34] where atoms leak from two trapped condensates and are detected in the output channels of a 50-50 beam splitter. Exact procedure for such state preparation is the subject of our future work

This work has been supported by CONACyT, EPSRC, the Royal Commission for the Exhibition of 1851 and the EU. 


\section{References}

[1] M. H. Anderson, J. R. Enser, M. R. Matthews, C. E. Wieman and E. Cornell, Science 269 (1995) 198.

[2] K. B. Davis, M.-O. Mewes, M. R. Andrews, N. J. van Druten, D. S. Durfee, D. M. Kurn, and W. Ketterle, Phys. Rev. Lett. 753969 (1995).

[3] S. M. Barnett, K. Burnett and J. A. Vaccaro, J. Res. Natl. Inst. Stand. Technol. 101 (1996) 593.

[4] M. R. Andrews, C. G. Townsend, H.-J. Miesner, D. S. Durfee, D. M. Kurn and W. Ketterle, Science 275 (1997) 637.

[5] B. P. Anderson and M. A. Kasevich, Science 282 (1998) 1686.

[6] D. S. Hall, M. R. Matthews, C. E. Wieman, and E. A. Cornell, Phys. Rev. Lett. 81 (1998) 1543.

[7] B. Yurke and D. Stoler, Phys. Rev. Lett. 57 (1986) 13.

[8] J. A. Dunningham and K. Burnett, J. Mod. Opt. 48 (2001) 1837.

[9] K. Burnett, S. Choi, M. Davis, J. A. Dunninghman, S. A. Morgan and M. Rusch, C. R. Acad. Sci. IV 2 (2001) 399.

[10] M. Lewenstein and L. You, Phys. Rev. Lett. 77 (1996) 3489.

[11] J. A. Dunningham, M. J. Collett and D. F. Walls, Phys. Lett. A 245 (1998) 49

[12] E. L. Bolda, S. M. Tan and D. Walls, Phys. Rev. A 57 (1998) 4686.

[13] D. S. Jin, J. R. Enscher, M.R. Matthews, C.E. Wieman and E.A. Cornell, Phys. Rev. Lett. 77 (1996) 420

[14] M.-O. Mewes, M. R. Andrews, N. J. van Druten, D.M. Kurn, D.S. Durfee, C.G. Townsend and W. Ketterle, Phys. Rev. Lett. 77 (1996) 988.

[15] Y. Lai and H. A. Haus, Phys. Rev. A 40 (1989) 854.

[16] J. Rogel-Salazar, G.H.C. New, S. Choi and K. Burnett, Phys. Rev. A 65 (2002) 023601 .

[17] W. P. Schleich, Quantum Optics in Phase-Space, (Wiley-VCH, Berlin 2001).

[18] S. Mancini and P. Tombesi, Europhys. Lett. 40 (1997) 351.

[19] D. T. Smithey, M. Beck, M. G. Raymer and A. Faridani, Phys. Rev. Lett. 70 (1993) 1244.

[20] Ch. Kurtsiefer, T. Pfau and J. Mlynek, Nature 386 (1997) 150.

[21] P. F. Byrd and M. D. Friedman, Handbook of Elliptic Integrals for Engineers and Scientists, (Springer, Berlin 1971). 
[22] W. Schleich, M. Pernigo, Fam Le Kien, Phys. Rev. A 44 (1991) 2172.

[23] K. Helmerson, D. Hutchinson, K. Burnett and W. D. Phillips, Physics World 12 (1999) 31.

[24] E. A. Burt, R. W. Ghirst, C. J. Myatt, M. J. Holland, E. A. Cornell and C. E. Wieman, Phys. Rev. Lett. 79 (1997) 337.

[25] P. O. Fedichev, M. W. Reynolds and G. V. Shlyapnikov, Phys. Rev. Lett. 77 (1996) 2921.

[26] D. M. Stamper-Kurn, H. -J. Miesner, A. P. Chikkatur, S. Inouye, J. Stenger and W. Keterle, Phys. Rev. Lett. 81 (1998) 2194.

[27] H. Fu and A. I. Solomon, J. Mod. Opt. 49 (2002) 259.

[28] A. Miranowicz, J. Bajer, M. R. B. Wahiddin and N. Imoto, J. Phys. A 34 (2001) 3887.

[29] D. Yao, Phys. Rev. A 55 (1997) 701.

[30] K. Wódkiewicz, P. L. Knight, S. J. Buckle and S. M. Barnett, Phys. Rev. A 35 (1987) 2567.

[31] B. Baseia, M. H. Y Moussa and V. S Bagnato, Phys. Lett. A 231 (1997) 331.

[32] S. M. Barnett and D. T. Pegg, Phys. Rev. Lett. 76 (1996) 4148.

[33] M. Dakna, J. Clausen, L. Knöll and D.-G. Welsch, Phys. Rev. A 59 (1999) 1658.

[34] Y. Castin and J. Dalibard, Phys. Rev. A 55 (1997) 4330. 